\title{
DETERMINAÇÃO DOS COEFICIENTES DOS HARMÔNICOS ESFÉRICOS PARA O CÁLCULO DOS PARÂMETROS PCV E PCO UTILIZANDO DADOS DE PROGRAMAS COMERCIAIS DE CALIBRAÇÃO
}

\author{
Determination of spherical harmonics coefficients for PCV and PCO parameters \\ estimation with commercial calibration software data \\ LUIZ FERNANDO MACEDO MORESCKI JUNIOR ${ }^{1,2}$ \\ LUIZ DANILO DAMASCENO FERREIRA ${ }^{1}$ \\ ${ }^{1}$ Universidade Federal do Paraná - UFPR \\ Curso de Pós-Graduação em Ciências Geodésicas - CPGCG \\ \{morescki, luizdanilo\}@ufpr.br \\ ${ }^{2}$ Instituto Federal de Educação, Ciência e Tecnologia de Santa Catarina - IFSC \\ Campus Jaraguá do Sul.
}

\begin{abstract}
RESUMO
Erros provenientes do centro de fase da antena, conhecidos como PCV - Phase Center Variations - são considerados agentes limitadores da acurácia do posicionamento relativo GNSS e do Posicionamento por Ponto Preciso (PPP). As PCVs dependem da direção do sinal do satélite e podem ser modeladas por parâmetros determinados por calibração de antenas em que são utilizados programas específicos, como por exemplo Wasoft/Kalib. Alguns autores afirmam que os programas aplicam funções harmônicas esféricas para a modelagem dos parâmetros PCV, mas não informam quais coeficientes são adotados para que a modelagem seja alcançada. Nesta pesquisa, são utilizados os parâmetros PCV de calibração determinados pelo programa comercial WaSoft/Kalib para a antena LEIAX 1202GG. O objetivo principal é calcular os coeficientes $a_{n m}$ e $b_{n m}$ da expansão em funções harmônicas esféricas. Os coeficientes são utilizados para determinar novos parâmetros PCV e PCO que são comparados aos parâmetros obtidos pelo programa comercial Wasoft/Kalib. Os novos parâmetros e os parâmetros calibrados são utilizados para determinar as coordenadas geodésicas de dois pontos com o objetivo de serem comparadas. Foram realizados testes para duas linhas de base diferentes e
\end{abstract}


os resultados confirmam diferenças submilimétricas para as PCVs e milimétricas para a componente vertical do vetor PCO.

Palavras-chave: Variações do Centro de Fase; Harmônicos Esféricos, WaSoft/Kalib.

\begin{abstract}
Errors from antenna phase center, known as PCV - Phase Center Variations - are considered a limiting agent of the relative GNSS positioning and the Precise Point Positioning (PPP) accuracy. The PCV are dependent of the direction of the satellite signal and can be modeled by parameters determined by antenna calibration, a whole process enclosing measurements but also specific softwares, for example WaSoft/Kalib. Authors state that some algorithms apply spherical harmonic functions to describe PCV parameters. But there are no certain information about which coefficients are taken in the modeling. In this research, PCV parameters of the LEIAX 1202GG antenna derived by the WaSoft/Kalib software are applied to an antenna calibration process. The main goal is to calculate spherical harmonic expansion coefficients, $a_{n m}$ and $b_{n m}$ to be applied on a new determination of PCV and PCO parameters. A comparison to the values obtained by a commercial software Wasoft/Kalib is carried out. The new and the original parameters are utilized to compute geodetic coordinates of two stations in order to be compared. Tests for two different base lines were realized. Sub-millimetric and millimetric deviations were found for PCV and for the UP component, respectively.
\end{abstract}

Keywords: Phase Center Variations; Spherical Harmonics; WaSoft/Kalib.

\title{
1. INTRODUÇÃO
}

Uma das aplicações dos sistemas de posicionamento por satélites é a determinação das coordenadas tridimensionais de pontos sobre a superfície da Terra com um nível de acurácia comparável ao. Para que tal qualidade no posicionamento possa ser atingida, é necessário o conhecimento e modelagem dos erros sistemáticos que afetam as medidas.

Nas medições efetuadas, as variações do centro de fase da antena se caracterizam como uma fonte de erros sistemáticos (FANG et al., 1998; MONICO, 2008; HUINCA, 2009; KRUEGER et al., 2012).

Segundo o IEEE (Institute of Electrical and Electronics Engineers), define-se centro de fase como:

A localização de um ponto associado a uma antena de forma tal que, se for tomado como o centro de uma esfera da qual o raio se estende ao campo distante, a fase de um dado componente do campo sobre a superfície da esfera de radiação é "essencialmente" constante, ao menos sobre a porção da superfície onde a radiação é significante.

Pelo teorema da reciprocidade, numa situação de recepção a antena apresenta características idênticas às apresentadas por ela numa situação de emissão 
(MARKOV, 1965; KRAUS, 1983; BALANIS, 2005). Desta forma, o centro de fase de uma antena, empregada na recepção de energia pode ser entendido como o ponto da antena em que o sinal é coletado. Seeber (2003) destaca que o posicionamento em navegação e em geodésia refere-se ao centro de fase elétrico o qual se diferencia do centro mecânico da antena que frequentemente coincide com a interseção do eixo de simetria vertical da antena com o plano de terra.

Görres et al. (2006) comentam que em aplicações geodésicas que necessitam de acurácia ao nível do milímetro é importante conhecer a exata posição do centro de fase da antena do receptor GNSS, uma vez que a posição é estimada, justamente, onde se encontra a antena.

Neste contexto, o problema é a instabilidade do centro de fase. Sua posição varia com a intensidade e direção (elevação sobretudo e azimute) dos sinais e é diferente para cada uma das ondas portadoras (MONICO, 2008).

O conhecimento completo do centro de fase elétrico da antena se dá mediante a determinação do PCO (Phase Center Offset) e das PCV (Phaser Center Variations). O PCO é o vetor com origem no ARP (ingl. Antenna Reference Point ponto de referência da antena) ao centro de fase elétrico médio. Segundo Freiberger Junior (2007), as PCVs expressam as alterações do estado da fase da onda portadora em dependência da direção do sinal incidente, o que é ilustrado pela Figura 1.

Figura 1 - Localização do ARP, vetor PCO, e das PCV. Fonte: Adaptado de Freiberger Junior (2007)

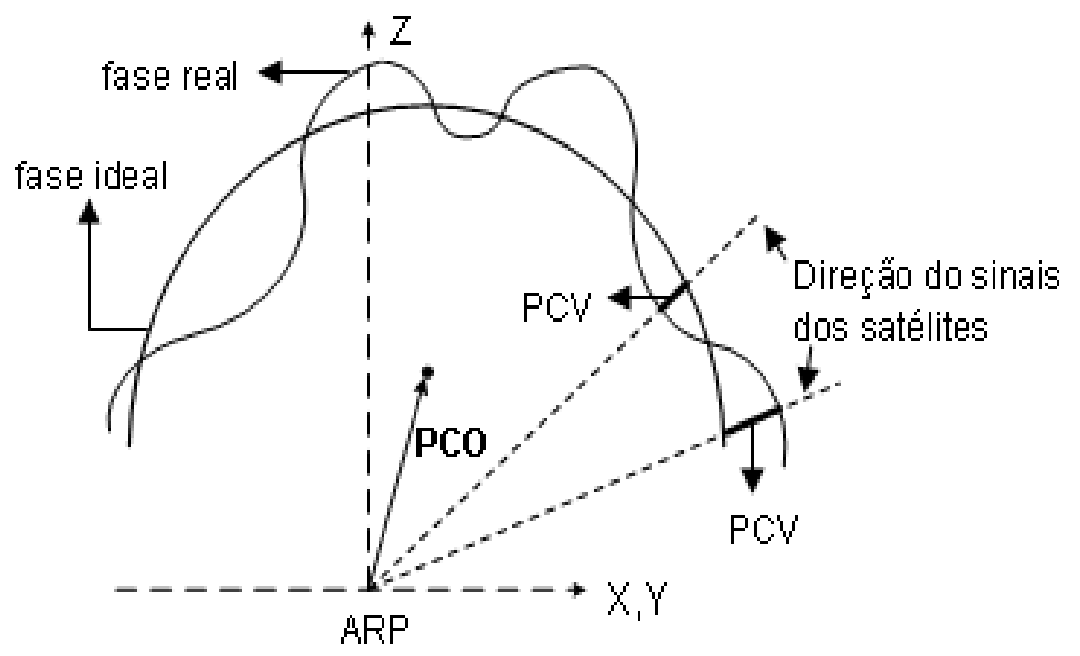

Nas aplicações GNSS que exigem alto nível de acurácia, a exemplo dos trabalhos que envolvem geodinâmica, uma das principais fontes de erros se 
estabelece na antena do receptor. Tratam-se dos erros ocasionados pelas variações do centro de fase da antena. Para aplicações que requerem acurácia, a posição do centro de fase de todas as antenas envolvidas no projeto tem que ser conhecida com exatidão (SEEBER, 2003). As PCVs participam de forma decisiva na determinação da componente vertical do posicionamento, causando variações que podem chegar à ordem de grandeza de centímetro. Em Mader (1999), comenta-se que a magnitude das variações pode atingir valores de até $10 \mathrm{~cm}$.

\section{CALIBRAÇÃO DE ANTENAS}

As PCV foram determinadas e analisadas em experimentos de laboratório desde o começo do desenvolvimento do sistema GNSS (SCHMITZ et al., 2002).

$\mathrm{O}$ crescente número de trabalhos que tratam das PCVs é um indício da importância que o tema exerce no âmbito das aplicações geodésicas que requerem acurácia da ordem de milímetros. Os valores determinados para as PCVs fazem com que os erros ocasionados na estimativa de coordenadas sejam minimizados ou mesmo eliminados por completo, quando utilizados no processamento dos dados.

No entanto, o método pelo qual esta determinação é alcançada, consiste em um processo de calibração das antenas dos receptores. Tal procedimento pode ser realizado por dois métodos: absoluto ou relativo; e em dois tipos de ambientes: câmaras anecóicas ou em campo. Segundo Menge (2003), a calibração de antenas GNSS pode ser alcançada pelas formas:

- determinação do PCO;

- calibração absoluta em ambientes controlados;

- calibração absoluta em campo e

- calibração relativa em campo.

Para mais detalhes sobre os métodos, Bartels (1997), Görres et al. (2006), Freiberger Junior (2007) e Huinca (2009) são indicados.

A calibração de antenas GNSS tem por objetivo a determinação dos parâmetros que expressam as coordenadas do vetor $\mathrm{PCO}$ e dos respectivos parâmetros PCV dependentes dos ângulos de azimute e elevação dos satélites observados. A determinação dos parâmetros é feita mediante a utilização de programas específicos, por exemplo, GNPCV, WaSoft/Kalib e Bernese. Neste processo, destaca-se a importância da BCAL/UFPR (Base de Calibração da Universidade Federal do Paraná), primeira na América Latina (Krueger et al. (2008), Huinca (2009) e Huinca et al. (2012)).

\subsection{Modelagem Matemática dos Parâmetros PCV}

Considerando os estudos de Rothacher et al. (1995) acerca do método de determinação dos parâmetros PCV utilizado pelo software Bernese 3.4, considera-se que o programa WaSoft/Kalib utilize a mesma modelagem matemática. Assim, o modelo matemático é escrito como: 


$$
p c v(\alpha, z)=\sum_{n=0}^{n_{m a ́ x}} \sum_{m=0}^{n} \tilde{P}_{n m}(\cos z)\left(a_{n m} \cos m \alpha+b_{n m} \operatorname{sen} m \alpha\right) \text {, }
$$

onde:

$\alpha=$ azimute do satélite, contado a partir do norte para leste, em graus.

$z=$ ângulo zenital do satélite, em graus.

$\tilde{P}_{n m}=\Lambda(n, m) P_{n m}=$ polinômios de Legendre de grau $n$ e ordem $m(m \leq n)$ normalizados.

$P_{n m}=$ polinômios de Legendre.

$\Lambda(n, m)=$ função normalizadora.

$a_{n m}$ e $b_{n m}=$ são os coeficientes dos harmônicos esféricos.

A normalização, proposta em Schaer et al. (1995), é dada por:

$$
\Lambda(n, m)=\left(2 \frac{2 n+1}{1+\delta_{0 m}} \frac{(n-m) !}{(n+m) !}\right)^{1 / 2}
$$

onde $\delta$ é o delta de Kronecker.

\section{MATERIAIS E METODOLOGIA}

Para esta pesquisa empregaram-se os parâmetros de calibração da antena LEIAX1202GG obtidos por campanhas de calibração relativas em campo realizadas nos dias 24, 27 de setembro e 01 de outubro de 2011 na BCAL/UFPR. O processamento dos dados para a determinação dos parâmetros de calibração foi conduzido no programa WaSoft/Kalib, em que se empregou a antena LEIAR25 como antena de referência. Ambas as antenas foram conectadas ao receptor Leica GPS 1200.

Neste trabalho, os parâmetros originários do programa WaSoft/Kalib foram representados por $\mathrm{PCV}_{\mathrm{ws}}$ e $\mathrm{PCO}_{\mathrm{ws}}$,

A proposta metodológica visa a determinação dos coeficientes $a_{n m}$ e $b_{n m}$ fazendo uso das relações 3 e 4 :

$$
\begin{aligned}
& a_{n m}=\int_{0}^{2 \pi} \int_{0}^{\pi} p \operatorname{cv}(\alpha, z) \cos (m \alpha) \tilde{P}_{n m}(\cos z) \operatorname{sen} z d z d \alpha \\
& b_{n m}=\int_{0}^{2 \pi} \int_{0}^{\pi} p c v(\alpha, z) \operatorname{sen}(m \alpha) \tilde{P}_{n m}(\cos z) \operatorname{sen} z d z d \alpha
\end{aligned}
$$

Para o cálculo das integrais duplas foi empregado o método numérico trapezoidal, para o qual Atkinson (1978) e Ralston e Rabinowitz (1984) são indicados. 
Evidentemente, o número de coeficientes $a_{n m}$ e $b_{n m}$ depende do grau $n$ e ordem $m$ relativos ao desenvolvimento da equação (1). A título de exemplo, para $n=m=2$ existem 6 coeficientes $a_{n m}$ e $3 b_{n m}$ a serem determinados, uma vez que os coeficientes $b_{n 0}$, não constam do desenvolvimento, como verificado Equação 1.

Os parâmetros PCV, dependentes de azimute e ângulo zenital, necessários para a determinação de $a_{n m}$ e $b_{n m}$ são dispostos em um arquivo de texto gerado pelo programa Wasoft/Kalib. Neste arquivo, as PCVs são organizadas em duas matrizes de dimensões 73 x 18, referentes às ondas portadoras L1 e L2. Em cada matriz as linhas correspondem ao valor do azimute em intervalos de $5^{\circ}$, com início em $0^{\circ} \mathrm{e}$ término em $360^{\circ}$, em quanto as colunas correspondem ao valor do ângulo zenital, no mesmo intervalo e início, mas término em $85^{\circ}$.

Assim, os coeficientes das funções harmônicas esféricas são utilizados na determinação de novos parâmetros PCV através da equação (1), os quais, para efeito deste trabalho, são denominados $\mathrm{PCV}_{\text {harm }}$.

\subsection{Determinação do Vetor PCO}

Os parâmetros $\mathrm{PCV}_{\text {harm }}$ devem ser vinculados a um vetor $\mathrm{PCO}_{\text {harm }}$ que indica a posição do centro de fase médio em relação ao ponto ARP da antena, tomado como origem. O vetor PCO é conhecido pelas suas componentes (N, E, U) - ingl. North, East, Up. Conforme Menge (2003), um conjunto de parâmetros PCO e PCV produz correções relativas ao ARP, segundo Menge (2003), da forma:

$$
\Delta(\alpha, z)=\boldsymbol{P C O} \cdot \boldsymbol{e}+\operatorname{PCV}(\alpha, z),
$$

onde $\boldsymbol{e}$ representa o vetor unitário na direção do satélite.

Rothacher et al. (1995) comentam que um conjunto original de correções pode ser transformado em um novo conjunto de correções, com um novo vetor PCO e suas correspondentes PCVs, que surtirão o mesmo efeito que o conjunto original em termos de resultados quando aplicados no processamento de dados GNSS.

Portanto, as correções obtidas com os parâmetros provenientes do programa Wasoft/Kalib podem ser utilizadas para a determinação de um vetor $\mathrm{PCO}_{\text {harm }}$ que esteja vinculado às $\mathrm{PCV}_{\text {harm. }}$.

Observando a disposição das PCVs em uma matriz de 73 linhas por 18 colunas, o produto escalar na equação (5) pode ser representado matricialmente por 


$$
\boldsymbol{P C O} \cdot \boldsymbol{e}=\left[\begin{array}{ccc}
\cos \alpha_{1} \operatorname{sen} z_{1} & \operatorname{sen} \alpha_{1} \operatorname{sen} z_{1} & \cos z_{1} \\
\cos \alpha_{2} \operatorname{sen} z_{1} & \operatorname{sen} \alpha_{2} \operatorname{sen} z_{1} & \cos z_{1} \\
\vdots & \vdots & \vdots \\
\cos \alpha_{73} \operatorname{sen} z_{1} & \operatorname{sen} \alpha_{73} \operatorname{sen} z_{1} & \cos z_{1} \\
\cos \alpha_{1} \operatorname{sen} z_{2} & \operatorname{sen} \alpha_{1} \operatorname{sen} z_{2} & \cos z_{2} \\
\vdots & \vdots & \vdots \\
\vdots & \vdots & \vdots \\
\cos \alpha_{73} \operatorname{sen} z_{18} & \operatorname{sen} \alpha_{73} \operatorname{sen} z_{18} & \cos z_{18}
\end{array}\right]\left[\begin{array}{l}
N \\
E \\
\end{array}\right]
$$

onde a primeira matriz do lado direito da equação pode ser considerada como matriz expansão hemisférica, [EH]. Assim, a equação (5) pode ser reescrita na forma:

$$
\left[\begin{array}{c}
\Delta\left(\alpha_{1}, z_{1}\right) \\
\Delta\left(\alpha_{2}, z_{1}\right) \\
\vdots \\
\Delta\left(\alpha_{73}, z_{1}\right) \\
\Delta\left(\alpha_{1}, z_{2}\right) \\
\vdots \\
\vdots \\
\Delta\left(\alpha_{73}, z_{18}\right)
\end{array}\right]=[E H]\left[\begin{array}{c}
N \\
E \\
U
\end{array}\right]+\left[\begin{array}{c}
P C V\left(\alpha_{1}, z_{1}\right) \\
P C V\left(\alpha_{2}, z_{1}\right) \\
\vdots \\
P C V\left(\alpha_{73}, z_{1}\right) \\
P C V\left(\alpha_{1}, z_{2}\right) \\
\vdots \\
\vdots \\
P C V\left(\alpha_{73}, z_{18}\right)
\end{array}\right] .
$$

Relacionando as correções provenientes do programa de calibração com os novos parâmetros $\mathrm{PCV}_{\text {harm: }}$ :

$$
\left[\begin{array}{l}
N \\
E \\
U
\end{array}\right]_{\text {harm }}=[E H]^{-1}\left[\begin{array}{ccc}
\Delta\left(\alpha_{1}, z_{1}\right) & - & P C V\left(\alpha_{1}, z_{1}\right) \\
\Delta\left(\alpha_{2}, z_{1}\right) & - & P C V\left(\alpha_{2}, z_{1}\right) \\
\Delta\left(\alpha_{73}, z_{1}\right) & - & P C V\left(\alpha_{73}, z_{1}\right) \\
\Delta\left(\alpha_{1}, z_{2}\right) & - & P C V\left(\alpha_{1}, z_{2}\right) \\
& \vdots & \\
\underbrace{\Delta\left(\alpha_{73}, z_{18}\right)}_{\text {correçồes ws }} & - & \text { parâmetros harm }
\end{array}\right] .
$$

Após a determinação dos parâmetros $\mathrm{PCV}_{\text {harm }}$ e $\mathrm{PCO}_{\text {harm }}$, foi gerado um arquivo digital em formato de texto para ser utilizado no processamento dos dados resultantes do posicionamento relativo estático das observações GNSS utilizando o programa Leica Geo Office. Uma taxa de gravação de 1 segundo foi utilizada para a obtenção dos dados, os quais foram processados com efemérides transmitidas e sem considerar modelos troposféricos. 
Com o objetivo de validar a proposta metodológica, foram comparadas as coordenadas geodésicas de dois pontos obtidas primeiramente a partir da inclusão de parâmetros do programa Wasoft/Kalib no processamento, e num segundo momento, a partir da inclusão de parâmetros $\mathrm{PCV}_{\text {harm }}$ e $\mathrm{PCO}_{\text {harm }}$. Ressalta-se que foram mantidas as mesmas características de processamento em ambas as estratégias, com exceção dos parâmetros do centro de fase acima citados.

Os pontos para os quais foram determinadas as coordenadas são denominados pilar 2000 e ponto 1, enquanto o ponto considerado como referência para o processamento relativo estático é denominado pilar 1000. Quanto à linha de base, o pilar 2000 e pilar 1000, pertencentes à BCAL/UFPR (Figura 2), possuem entre si uma linha de base de aproximadamente $6 \mathrm{~m}$, enquanto o ponto 1 possui uma linha de base de aproximadamente $100 \mathrm{~km}$.

Figura 2 - Pilares 1000 e 2000 da BCAL/UFPR. Fonte: Adaptado de Freiberger
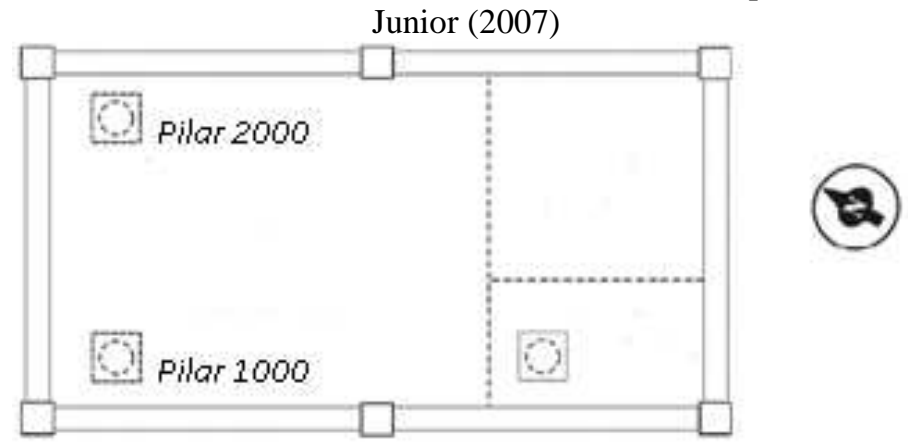

\section{DADOS}

Os parâmetros PCV determinados pelo programa de calibração originaram dois grupos de dados: as PCVs referentes ao dia 24 de setembro, denominadas $\mathrm{PCV}_{\text {ws_24 }} \mathrm{e}$ as PCVs resultantes da média aritmética das PCVs referentes aos três dias de calibração $\left(24,27 / 09\right.$ e 01/10/2011), representadas por $\mathrm{PCV}_{\text {ws_med. }}$

\section{RESULTADOS}

Nesta seção são apresentados os resultados da aplicação da proposta metodológica a cada grupo de dados. Evidentemente, a determinação dos parâmetros $\mathrm{PCV}_{\text {harm }}$ está relacionada aos coeficientes dos harmônicos esféricos, cujo número total depende do grau e ordem do desenvolvimento a que são submetidos. Diante dos testes realizados, os melhores resultados para as $\mathrm{PCV}_{\text {harm }}$ foram obtidos para $\mathrm{n}=\mathrm{m}=16$.

Como a pesquisa utiliza dois conjuntos de dados, e a fim de proceder a análise separadamente, são apresentados os resultados da aplicação das $\mathrm{PCV}_{\text {ws_24 }} \mathrm{e}$ das $\mathrm{PCV}_{\text {ws_med }}$ nas seções 5.1 e 5.2, respectivamente. 


\subsection{Resultados Considerando os Dados $\mathbf{P C V}_{\text {ws } 24}$}

Pelas equações (2) e (3) determinam-se os coeficientes das funções harmônicas esféricas, levando-se em conta $\mathrm{n}_{\max }=16$. Desta forma, as $P C V_{\text {harm_24, }}$, dependentes de azimute e elevação, são determinadas com o emprego da equação (1).

Os resultados das $\mathrm{PCV}_{\text {harm_24 }}$ relativas à onda portadora $\mathrm{L} 1$ são apresentados no gráfico da Figura 3.

Figura 3 - Parâmetros PCV harm_24 $_{-}$L1.

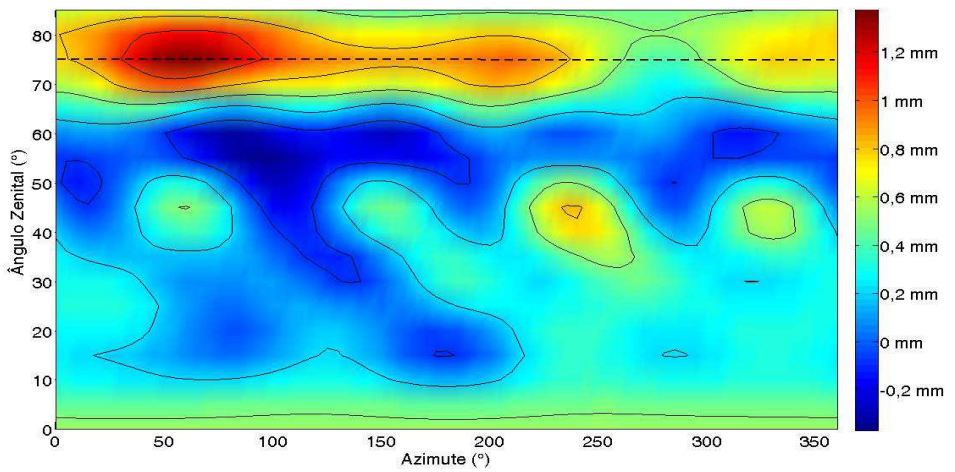

Nota-se que os menores valores das PCVs estão entre os ângulos zenitais de $50^{\circ}$ e $60^{\circ}$, associados aos ângulos azimutais próximos a $80^{\circ}$. Os maiores valores são verificados próximos ao ângulo zenital de $75^{\circ}$, entre os azimutes $50^{\circ}$ e $100^{\circ}$. A linha tracejada, também apresentada no gráfico, faz referência ao ângulo de corte zenital de $75^{\circ}$, que corresponde à máscara de elevação de $15^{\circ}$ adotada no processamento relativo estático executado a posteriori.

As $\mathrm{PCV}_{\text {harm_24 }}$ referentes à onda portadora L2 são representadas pelo gráfico da Figura 4.

Figura 4 - Parâmetros PCV $\mathrm{V}_{\text {harm_24 }}$ - L2.

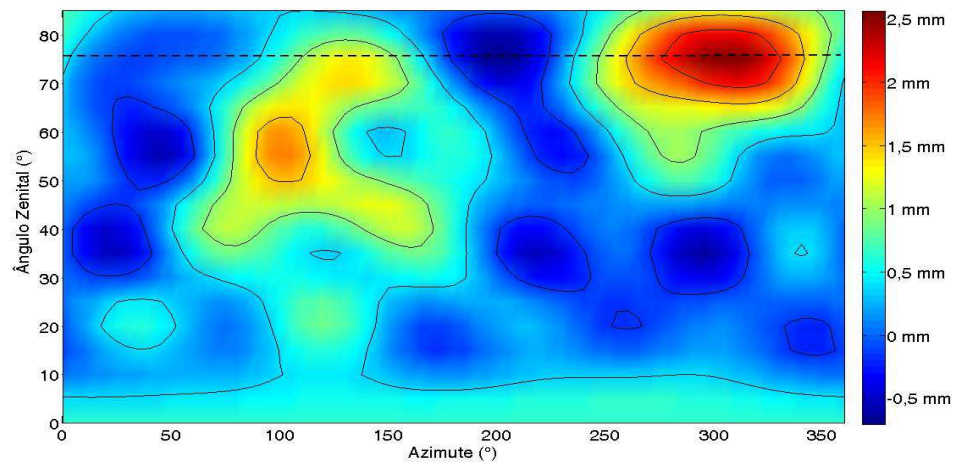

Bol. Ciênc. Geod., sec. Artigos, Curitiba, v. 20, no 2, p.444-466, abr-jun, 2014. 
Observa-se que tanto os mínimos como os máximos valores das PCVs estão próximos ao ângulo zenital de $75^{\circ}$. Com relação ao ângulo azimutal, as menores PCVs são encontradas entre os azimutes $150^{\circ}$ e $200^{\circ}$, enquanto as maiores próximas ao azimute $300^{\circ}$.

Os valores PCV $_{\text {harm_24 }}$ para as ondas portadoras L1 e L2 devem estar associados a um centro de fase médio localizado pelo vetor $\mathrm{PCO}_{\text {harm_24 }}$, cujas componentes estão representadas na Tabela 1 .

Tabela 1 - Componentes do vetor $\mathrm{PCO}_{\text {harm_24 }}-\mathrm{L} 1$ e L2.

\begin{tabular}{c|c|c}
\hline Componente & PCO $_{\text {harm_24 }}-\mathbf{L 1}$ & PCO $_{\text {harm_24 }}-\mathbf{L 2}$ \\
\hline $\mathrm{N}$ & $0,99 \mathrm{~mm}$ & $-0,60 \mathrm{~mm}$ \\
\hline $\mathrm{E}$ & $-0,95 \mathrm{~mm}$ & $1,29 \mathrm{~mm}$ \\
\hline $\mathrm{U}$ & $63,25 \mathrm{~mm}$ & $61,47 \mathrm{~mm}$ \\
\hline
\end{tabular}

As diferenças entre os parâmetros $\mathrm{PCV}_{\text {harm_24 }}$ e $\mathrm{PCV}_{\text {ws_24 }}$ relativos à onda portadora L1 são representadas pelo gráfico da Figura 5.

Figura 5 - Diferenças entre $\mathrm{PCV}_{\text {harm_24 }} \mathrm{ePCV}_{\mathrm{ws} \_24}-\mathrm{L} 1$.

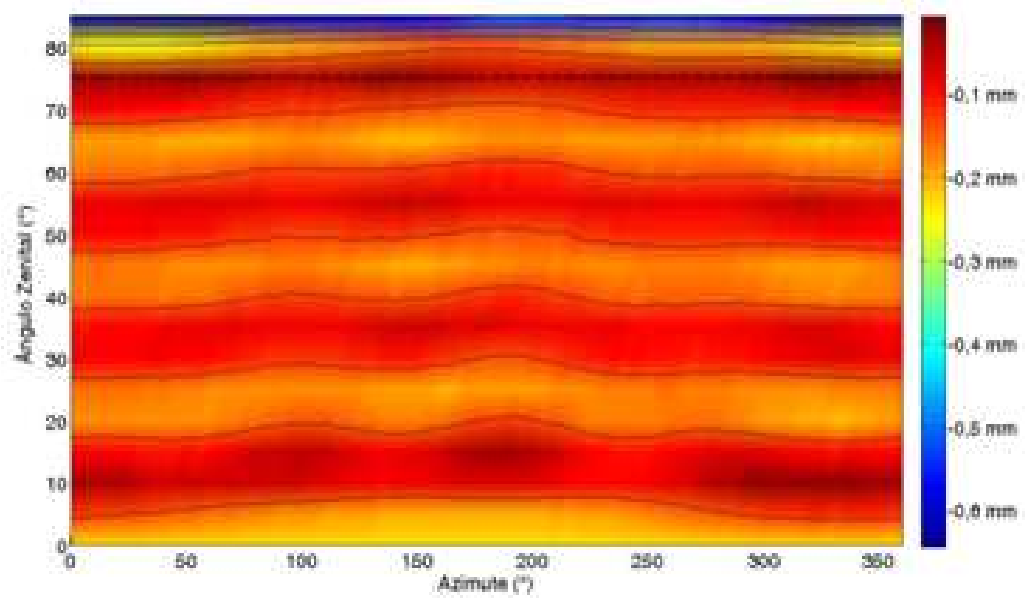

Nota-se que as maiores diferenças, em módulo, se verificam em ângulos zenitais superiores a $80^{\circ}$. Considerando os ângulos zenitais até $75^{\circ}$, as diferenças alcançadas possuem um valor máximo, em módulo, de $0,3 \mathrm{~mm}$, na região entre $0^{\circ} \mathrm{e}$ $10^{\circ}$. As mínimas diferenças chegam a zero para ângulo zenital de $10^{\circ}$ e azimutes próximos a $300^{\circ}$.

As diferenças entre os parâmetros $\mathrm{PCV}_{\text {harm_24 }}$ e os parâmetros $\mathrm{PCV}_{\mathrm{ws} \_24}$ relativos à onda portadora L2 estão representadas no gráfico da Figura 6. 
Figura 6 - Diferenças entre PCV $\mathrm{Parm} \_24_{\text {e PCV }} \mathrm{PC}_{\text {ws_24 }}-\mathrm{L} 2$.

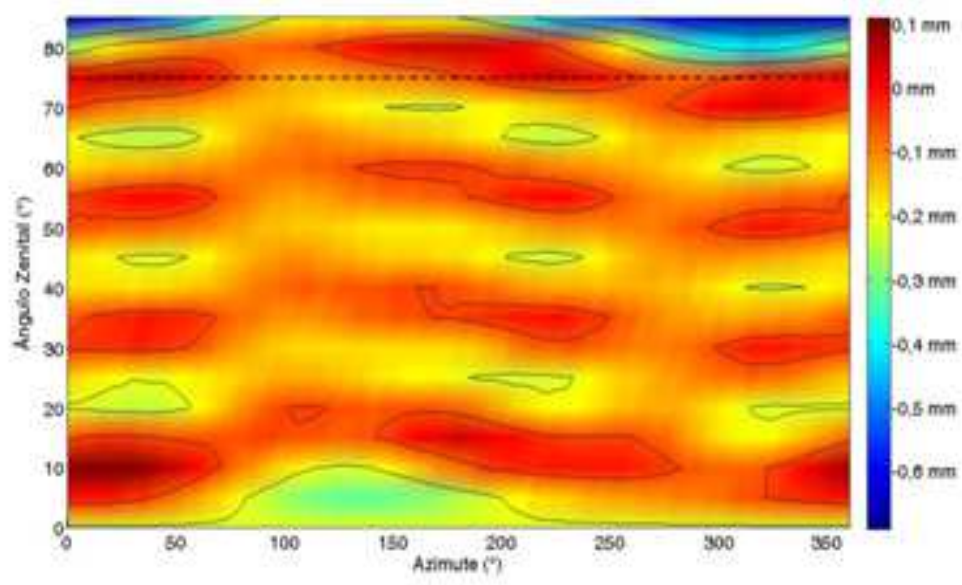

Constata-se que as maiores discrepâncias, em módulo, estão relacionadas aos ângulos zenitais superiores a $75^{\circ}$ e valem aproximadamente $0,7 \mathrm{~mm}$. Considerando o intervalo zenital de $0^{\circ}$ a $75^{\circ}$, as maiores diferenças, em módulo, ocorrem nos intervalos de $0^{\circ}$ a $10^{\circ}$ e de $100^{\circ}$ a $180^{\circ}$, e valem cerca de $0,3 \mathrm{~mm}$.

A comparação entre os parâmetros $\mathrm{PCO}_{\text {harm }}$ e $\mathrm{PCO}_{\mathrm{ws}}$, para a campanha de 24/09/2011, também foi efetuada. As diferenças entre os valores das componentes

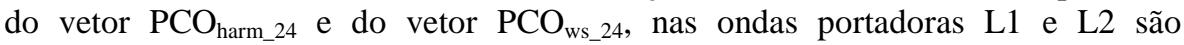
apresentadas na Tabela 2.

Tabela 2 - Diferença entre as componentes dos vetores $\mathrm{PCO}_{\text {harm } \_24}$ e $\mathrm{PCO}_{\text {ws_24 }}-\mathrm{L} 1 \mathrm{e}$ L2.

\begin{tabular}{c|c|c}
\hline $\begin{array}{c}\text { Diferença } \\
\text { Componentes }\end{array}$ & $\begin{array}{c}\text { Onda } \\
\text { Portadora L1 }\end{array}$ & $\begin{array}{c}\text { Onda } \\
\text { Portadora L2 }\end{array}$ \\
\hline $\mathrm{N}_{\text {harm_24 }}-\mathrm{N}_{\mathrm{ws} 224}$ & $-0,04 \mathrm{~mm}$ & $-0,05 \mathrm{~mm}$ \\
\hline $\mathrm{E}_{\text {harm_24 }}-\mathrm{E}_{\mathrm{ws} \_24}$ & $0,01 \mathrm{~mm}$ & $0,02 \mathrm{~mm}$ \\
\hline $\mathrm{U}_{\text {harm_24 }}-\mathrm{U}_{\mathrm{ws} \_24}$ & $0,14 \mathrm{~mm}$ & $0,11 \mathrm{~mm}$ \\
\hline
\end{tabular}

Após a determinação dos parâmetros $\mathrm{PCV}_{\text {harm_24 }} \mathrm{e}$ de seu vetor correspondente $\mathrm{PCO}_{\text {harm } 24}$, gerou-se um arquivo digital destes dados em formato de texto com o objetivo de ser aplicado no processamento relativo estático das coordenadas do pilar 2000.

A proposta de validação do método compreende estabelecer a comparação entre as coordenadas geodésicas dos pontos (pilar 2000 e ponto 1) quando obtidas com a aplicação de dois conjuntos distintos de parâmetros para o centro de fase da antena: parâmetros provenientes do programa de calibração, $\mathrm{PCV}_{\mathrm{ws}}$ e $\mathrm{PCO}_{\mathrm{ws}}$, e os parâmetros $\mathrm{PCV}_{\text {harm }}$ e $\mathrm{PCO}_{\text {harm }}$. 
Após o processamento relativo estático, as coordenadas do pilar 2000 e do ponto 1 obtidas com a utilização dos parâmetros $\mathrm{PCV}_{\text {harm_24 }}$ e $\mathrm{PCO}_{\text {harm_24, são }}$ comparadas com as coordenadas obtidas com os parâmetros $\mathrm{PCV}_{\text {ws_24 }} \mathrm{e} \mathrm{PCO}_{\text {ws_24. }} \mathrm{O}$ resultado da comparação para o pilar 2000 é apresentado na Tabela 3.

Tabela 3 - Diferenças entre as coordenadas do Pilar 2000 obtidas com parâmetros harm_24 e ws_24.

\begin{tabular}{c}
\hline $\begin{array}{c}\text { Diferenças Entre Coordenadas } \\
\text { Pilar 2000 }\end{array}$ \\
\hline$\Delta$ Latitude $=0$ \\
\hline$\Delta$ Longitude $=0$ \\
\hline$\Delta$ Altitude $=-1 \times 10^{-4} \mathrm{~m}$ \\
\hline
\end{tabular}

Infere-se que as coordenadas referentes à latitude e longitude geodésicas do pilar 2000 resultam iguais considerando resolução ao centésimo do milésimo de segundo de arco, quando aplicado tanto o conjunto de parâmetros $\mathrm{PCV}_{\text {harm_24 }} \mathrm{e}$ $\mathrm{PCO}_{\text {harm_24 }}$ quanto o conjunto $\mathrm{PCV}_{\text {ws_24 }}$ e $\mathrm{PCO}_{\text {ws_24. Nota-se que a diferença entre os }}$ valores da altitude do pilar 2000 corresponde a um décimo de milímetro.

A Tabela 4 apresenta a comparação entre as coordenadas do ponto 1 obtidas com os parâmetros $\mathrm{PCV}_{\text {harm_24 }} \mathrm{e} \mathrm{PCO}_{\text {harm_24 }} \mathrm{e}$ as obtidas com os parâmetros $\mathrm{PCV}_{\text {ws_24 }}$ e $\mathrm{PCO}_{\text {ws } 24}$

Tabela 4 - Diferenças entre as coordenadas do Ponto 1 obtidas com parâmetros harm_24 e ws_24.

\begin{tabular}{c}
\hline Diferenças Entre Coordenadas \\
Ponto 1 \\
\hline$\Delta$ Latitude $=0$ \\
\hline$\Delta$ Longitude $=0$ \\
\hline$\Delta$ Altitude $=-2 \times 10^{-4} \mathrm{~m}$ \\
\hline
\end{tabular}

Percebe-se que os valores referentes à latitude e longitude geodésicas mostram-se sem alteração para a resolução ao centésimo do milésimo de segundo de arco quando os dois conjuntos de parâmetros são aplicados. Na comparação da coordenada altitude, nota-se que a diferença alcança a ordem de grandeza de décimos de milímetros.

\subsection{Resultados Considerando os Dados $P C V_{\text {ws_med }}$}

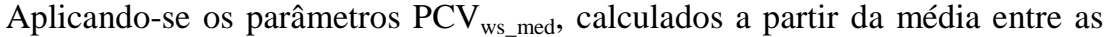
PCVs estabelecidas pelo programa WaSoft/Kalib para as campanhas de calibração da antena LEIAX 1202GG nos dias 24, 27/09 e 01/10/2011, foram determinados os coeficientes das funções harmônicas esféricas, pelas equações (2) e (3) considerando $\mathrm{n}_{\max }=16$ com o objetivo de determinar as $\mathrm{PCV}_{\text {harm_med }}$ por meio da equação (1). 
$\mathrm{O}$ gráfico da Figura 7, ilustra os resultados das $\mathrm{PCV}_{\text {harm_med }}$ referentes à onda portadora L1.

Figura 7 - Parâmetros $\mathrm{PCV}_{\text {harm_med }}-\mathrm{L} 1$.

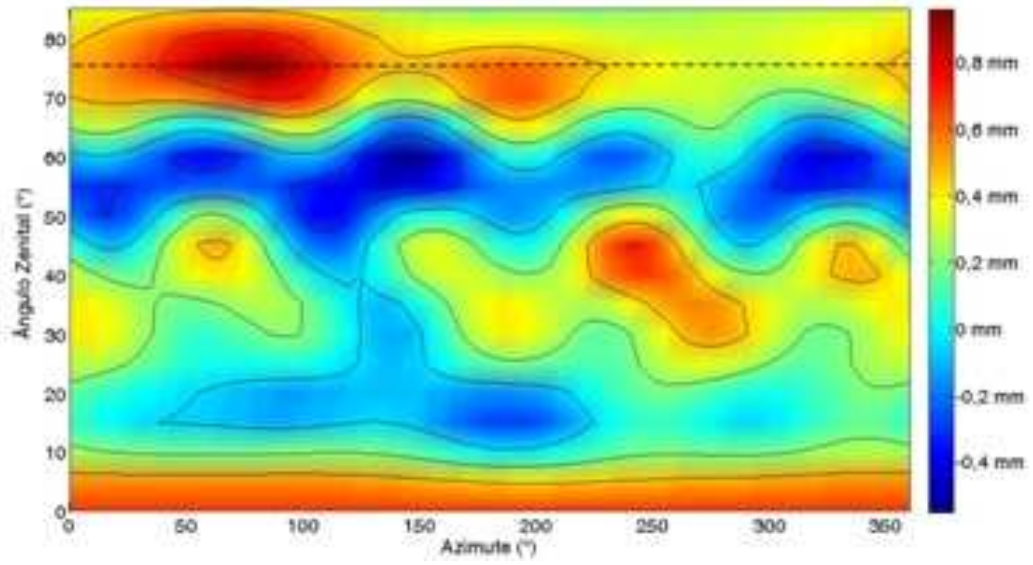

Nota-se que as menores $\mathrm{PCV}_{\text {harm_med }}$ estão associadas a ângulos zenitais próximos a $60^{\circ}$ e a azimutes próximos a $140^{\circ}$, enquanto que as maiores $\mathrm{PCV}_{\text {harm_med }}$ relacionam-se a ângulos zenitais próximos a $75^{\circ}$, entre os azimutes $50^{\circ}$ e $100^{\circ}$.

Os parâmetros $\mathrm{PCV}_{\text {harm_med }}$ relativos à onda portadora L2 estão apresentados no gráfico da Figura 8.

Figura 8 - Parâmetros $\mathrm{PCV}_{\text {harm_med }}-\mathrm{L} 2$.

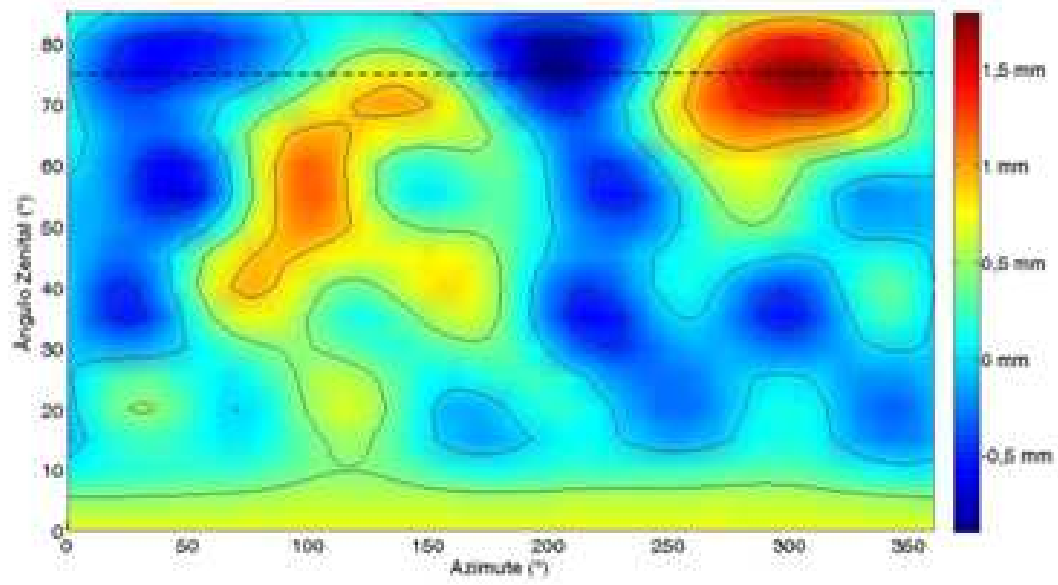

Bol. Ciênc. Geod., sec. Artigos, Curitiba, v. 20, nº 2, p.444-466, abr-jun, 2014. 
Observa-se que os menores valores são encontrados entre os ângulos zenitais de $75^{\circ}$ e $80^{\circ}$, próximos ao azimute de $200^{\circ}$, ao passo que os maiores valores apresentam-se nos ângulos zenitais próximos a $75^{\circ}$, em azimutes próximos a $300^{\circ}$.

Os valores das componentes do vetor $\mathrm{PCO}_{\text {harm_med }}$ relativos às ondas portadoras L1 e L2 são apresentados na Tabela 5.

Tabela 5 - Componentes $\mathrm{PCO}_{\text {harm_med }}-\mathrm{L} 1$ e L2.

\begin{tabular}{c|c|c}
\hline Componente & PCO $_{\text {harm_med }}-\mathbf{L 1}$ & PCO $_{\text {harm_med }}-\mathbf{L 2}$ \\
\hline $\mathrm{N}$ & $1,19 \mathrm{~mm}$ & $-0,42 \mathrm{~mm}$ \\
\hline $\mathrm{E}$ & $-0,92 \mathrm{~mm}$ & $0,70 \mathrm{~mm}$ \\
\hline $\mathrm{U}$ & $63,37 \mathrm{~mm}$ & $62,68 \mathrm{~mm}$ \\
\hline
\end{tabular}

As comparações realizadas entre os conjuntos $\mathrm{PCV}_{\text {harm_med }}$ e $\mathrm{PCO}_{\text {harm_med }}$ consistem da diferença algébrica entre eles e dos parâmetros provenientes do programa WaSoft/Kalib referentes a cada campanha de calibração considerada (dias 24, 27/09 e 01/10/2011).

O gráfico da Figura 9 apresenta as diferenças encontradas entre os parâmetros $\mathrm{PCV}_{\text {harm_med }}$ e os parâmetros $\mathrm{PCV}_{\text {ws_24 }}$ relativos à onda portadora L1.

Figura 9 - Diferenças entre PCV $\mathrm{Parm}_{\text {_med }}$ e $\mathrm{PCV}_{\text {ws_24 }}-\mathrm{L} 1$.

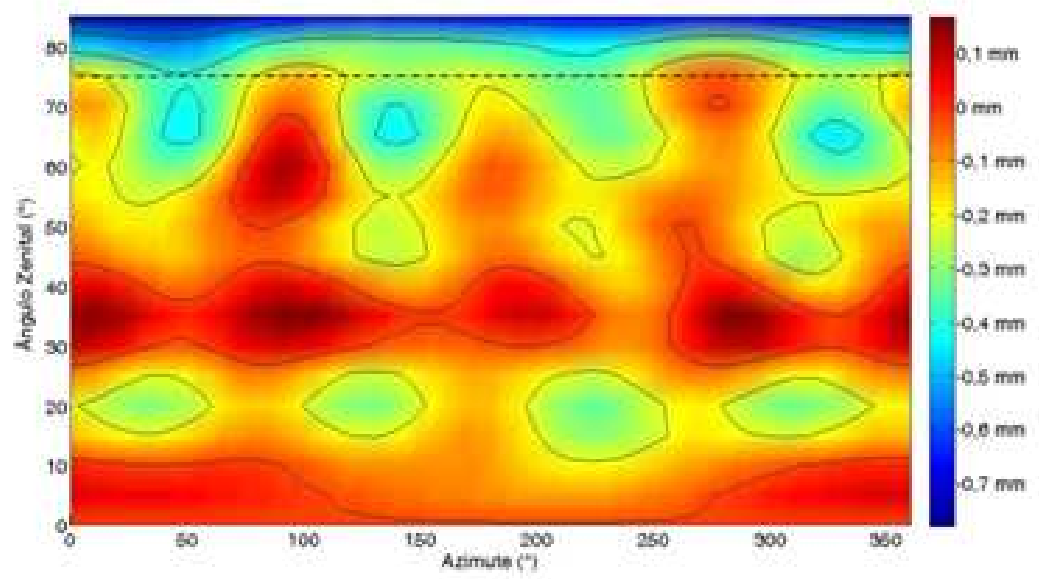

Observa-se que as maiores diferenças, em módulo, encontram-se em ângulos maiores que $75^{\circ}$, portanto, acima do ângulo zenital de corte. As diferenças máximas, próximas a $0,5 \mathrm{~mm}$, em módulo, são apresentadas no intervalo zenital de $60^{\circ}$ a $70^{\circ}$, associadas aos azimutes próximos a $50^{\circ}, 135^{\circ}$ e $335^{\circ}$.

No gráfico da Figura 10, apresentam-se as diferenças entre as $\mathrm{PCV}_{\text {harm_med }}$ e as $\mathrm{PCV}_{\text {ws_24 }}$ para a onda portadora L2. 
Figura 10 - Diferenças entre $\mathrm{PCV}_{\text {harm_med }}$ e $\mathrm{PCV}_{\mathrm{ws} \_24}-\mathrm{L} 2$.

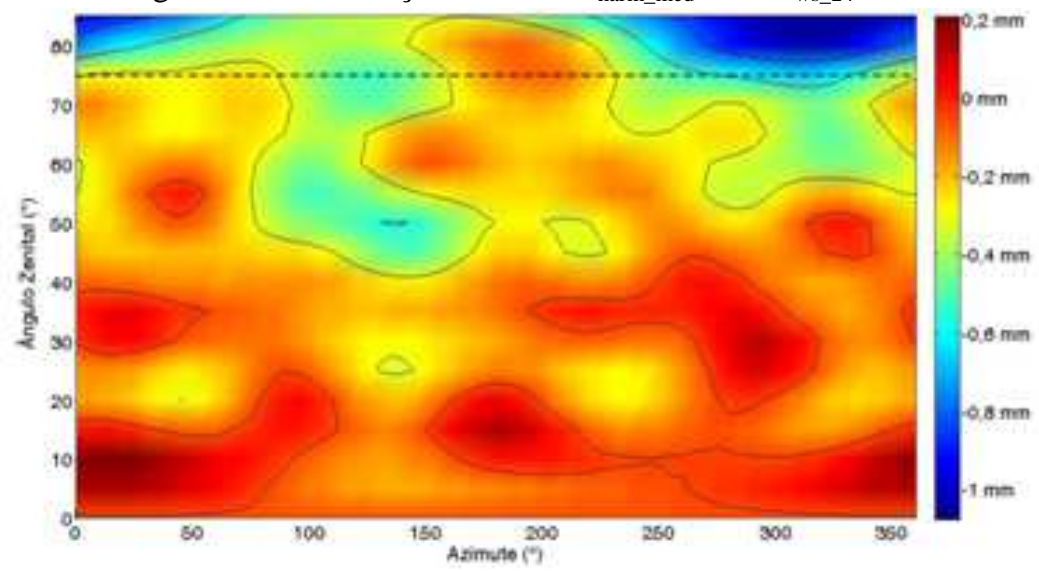

Considerando ângulos zenitais inferiores a $75^{\circ}$, as diferenças máximas apresentam o valor, em módulo, de $0,6 \mathrm{~mm}$, para ângulos zenitais próximos a $50^{\circ} \mathrm{e}$ azimutes próximos a $140^{\circ}$.

As diferenças entre as $\mathrm{PCV}_{\text {harm_med }}$ e as $\mathrm{PCV}_{\text {ws_27 }}$ para a onda portadora L1, são apresentadas no gráfico da Figura 11.

Figura 11 - Diferencas entre $\mathrm{PCV}_{\mathrm{harm} \text { med }}$ e $\mathrm{PCV}_{\mathrm{wc}}{ }_{77}-\mathrm{L} 1$.

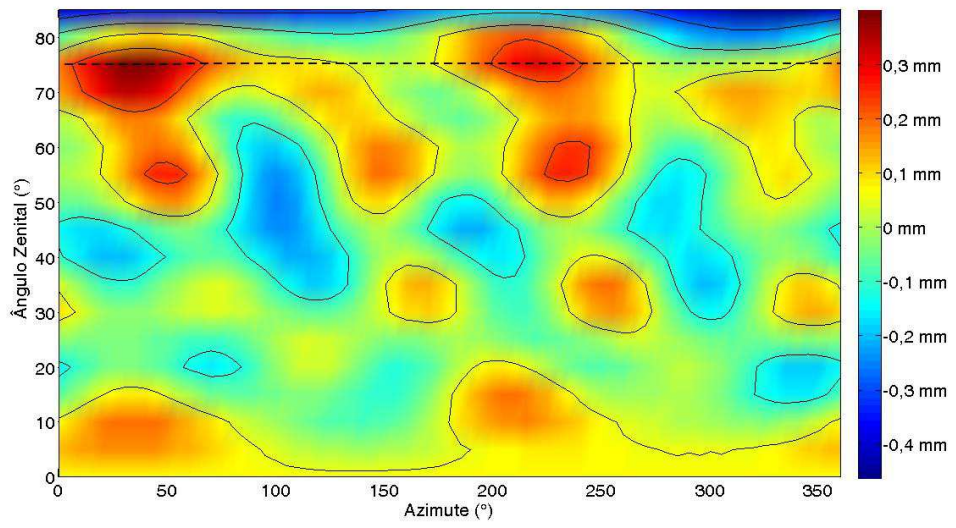

Considerando ângulos zenitais inferiores a $75^{\circ}$, as diferenças máximas, de $0,5 \mathrm{~mm}$, em módulo, encontram-se entre os ângulos $70^{\circ} \mathrm{e} 74^{\circ}$, em azimutes entre $0^{\circ}$ e $50^{\circ}$. 
Morescki, L. F. M. ; Ferreira, L. D. D.

As diferenças entre as $\mathrm{PCV}_{\text {harm_med }}$ e as $\mathrm{PCV}_{\text {ws_27 }}$ relativas à onda portadora L2 são mostradas no gráfico da Figura 12.

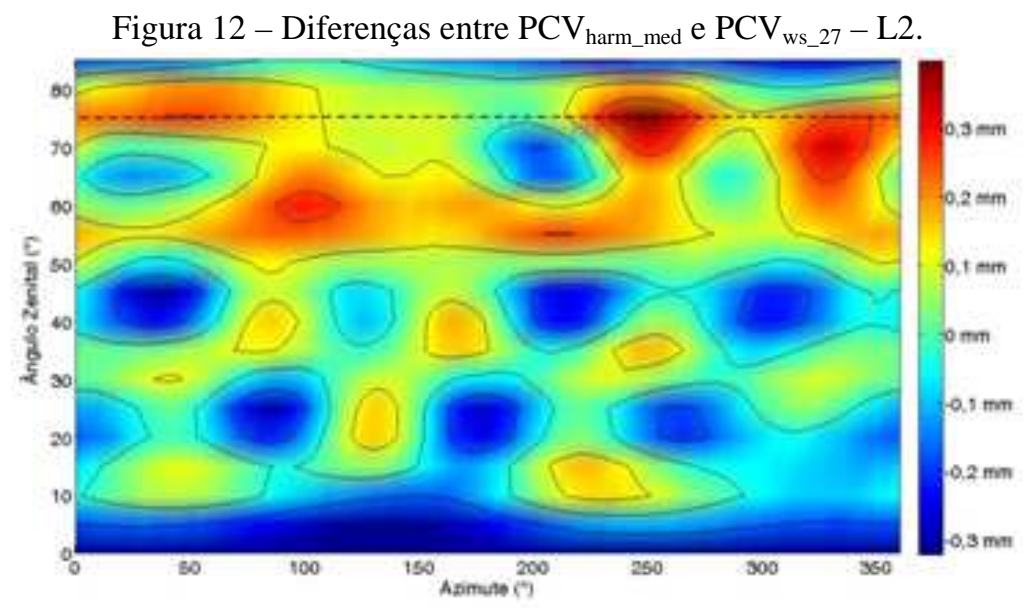

Considerando ângulos zenitais inferiores a $75^{\circ}$, o valor máximo para as diferenças, aproximadamente $0,3 \mathrm{~mm}$, em módulo, é constatado para o ângulo zenital de $5^{\circ}$, entre $100^{\circ}$ e $160^{\circ}$ de azimute.

Confrontando as $\mathrm{PCV}_{\text {harm_med }} \mathrm{e}$ as $\mathrm{PCV}_{\mathrm{ws} \_01}$, relativas à onda portadora $\mathrm{L} 1$, são produzidas as diferenças como mostra o gráfico da Figura 13.

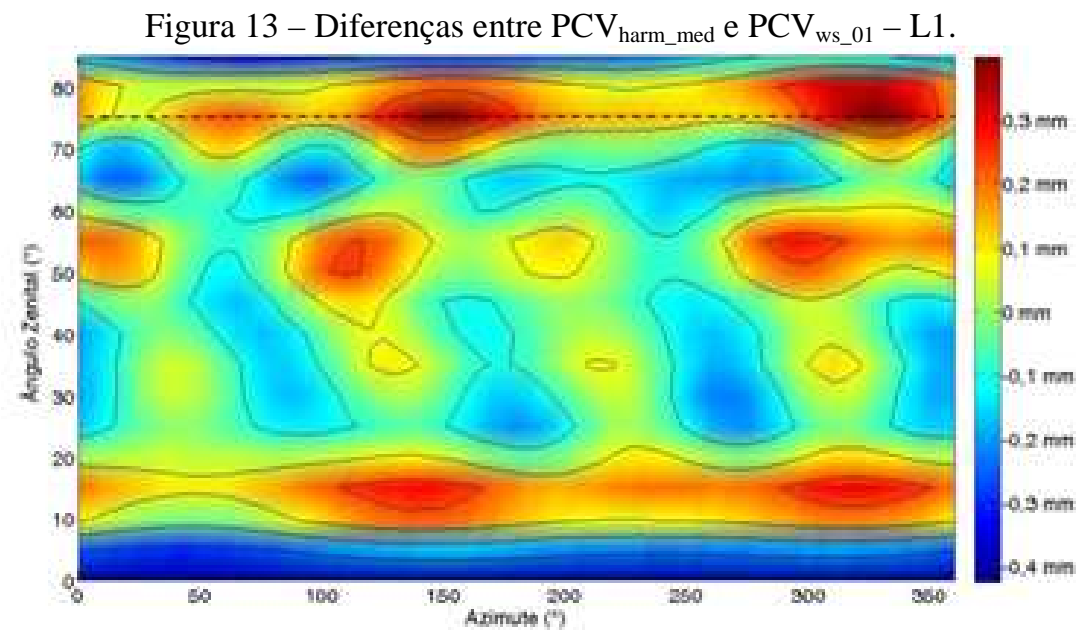

Bol. Ciênc. Geod., sec. Artigos, Curitiba, v. 20, nº 2, p.444-466, abr-jun, 2014. 
Observa-se que a máxima diferença, em módulo, considerando ângulos inferiores ao ângulo zenital de corte, vale $0,5 \mathrm{~mm}$ e é encontrada na direção do zênite $\left(0^{\circ}\right)$.

No gráfico da Figura 14 são apresentadas as diferenças entre as $\mathrm{PCV}_{\text {harm_med }} \mathrm{e}$ as $\mathrm{PCV}_{\text {ws_01 }}$ para a onda portadora $\mathrm{L} 2$.

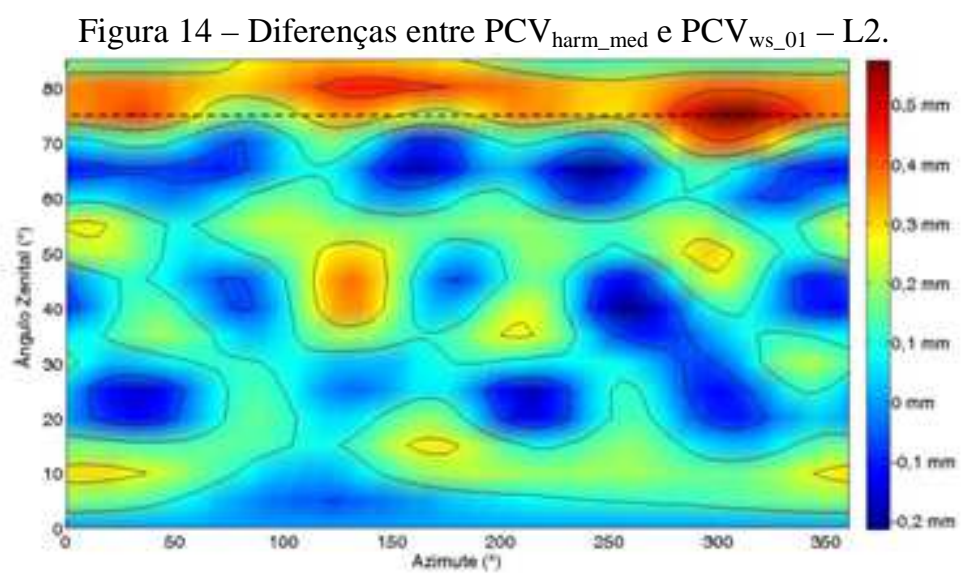

Considerando ângulos zenitais entre sua origem até o ângulo de corte, verificase que o módulo da máxima diferença vale $0,4 \mathrm{~mm}$ e está compreendida no intervalo entre os ângulos $40^{\circ}$ e $50^{\circ}$, correspondendo ao azimute de $125^{\circ}$.

Comparam-se também os valores dos parâmetros $\mathrm{PCO}_{\text {harm_med }}$ com os vetores PCO determinados pelo programa Wasoft/Kalib referentes aos dias 24, 27/09 e 01/10/2011. As diferenças entre as componentes do vetor $\mathrm{PCO}_{\text {harm_med }}$ e as do vetor $\mathrm{PCO}_{\text {ws_24 }}$, relativas às ondas portadoras L1 e L2, são mostradas na Tabela 6.

Tabela 6 - Diferenças entre as componentes dos vetores $\mathrm{PCO}_{\text {harm_med }} \mathrm{e}$ $\mathrm{PCO}_{\text {ws_24 }}-\mathrm{L} 1$ e L2.

\begin{tabular}{c|c|c}
\hline $\begin{array}{c}\text { Diferença } \\
\text { Componentes }\end{array}$ & $\begin{array}{c}\text { Onda } \\
\text { Portadora L1 }\end{array}$ & $\begin{array}{c}\text { Onda } \\
\text { Portadora L2 }\end{array}$ \\
\hline $\mathrm{N}_{\text {harm_med }}-\mathrm{N}_{\mathrm{ws} \_24}$ & $0,16 \mathrm{~mm}$ & $0,13 \mathrm{~mm}$ \\
\hline $\mathrm{E}_{\text {harm_med }}-\mathrm{E}_{\mathrm{ws} \_24}$ & $0,04 \mathrm{~mm}$ & $-0,57 \mathrm{~mm}$ \\
\hline $\mathrm{U}_{\text {harm_med }}-\mathrm{U}_{\mathrm{ws} \_24}$ & $0,26 \mathrm{~mm}$ & $1,32 \mathrm{~mm}$ \\
\hline
\end{tabular}

A Tabela 7 apresenta as diferenças entre as componentes do vetor $\mathrm{PCO}_{\text {harm_med }}$ e as do vetor $\mathrm{PCO}_{\text {ws_27 }}$ para as ondas portadoras L1 e L2. 
Tabela 7 - Diferenças entre as componentes dos vetores $\mathrm{PCO}_{\text {harm_med }} \mathrm{e}$ $\mathrm{PCO}_{\text {ws } 27}-\mathrm{L} 1$ e L2.

\begin{tabular}{c|c|c}
\hline $\begin{array}{c}\text { Diferença } \\
\text { Componentes }\end{array}$ & $\begin{array}{c}\text { Onda } \\
\text { Portadora L1 }\end{array}$ & $\begin{array}{c}\text { Onda } \\
\text { Portadora L2 }\end{array}$ \\
\hline $\mathrm{N}_{\text {harm_med }}-\mathrm{N}_{\text {ws_27 }}$ & $-0,1 \mathrm{~mm}$ & $-0,01 \mathrm{~mm}$ \\
\hline $\mathrm{E}_{\text {harm_med }}-\mathrm{E}_{\mathrm{ws} \_27}$ & $-0,03 \mathrm{~mm}$ & $0,2 \mathrm{~mm}$ \\
\hline $\mathrm{U}_{\text {harm_med }}-\mathrm{U}_{\text {ws_27 }}$ & $0,78 \mathrm{~mm}$ & $-0,02 \mathrm{~mm}$ \\
\hline
\end{tabular}

As diferenças entre as componentes do vetor $\mathrm{PCO}_{\text {harm_med }}$ e as componentes do vetor $\mathrm{PCO}_{\text {ws_01 }}$ são apresentadas na Tabela 8 .

Tabela 8 - Diferenças entre as componentes dos vetores $\mathrm{PCO}_{\text {harm_med }} \mathrm{e}$ $\mathrm{PCO}_{\text {ws_01 }}-\mathrm{L} 1$ e L2.

\begin{tabular}{c|c|c}
\hline $\begin{array}{c}\text { Diferença } \\
\text { Componentes }\end{array}$ & $\begin{array}{c}\text { Onda } \\
\text { Portadora L1 }\end{array}$ & $\begin{array}{c}\text { Onda } \\
\text { Portadora L2 }\end{array}$ \\
\hline $\mathrm{N}_{\text {harm_med }}-\mathrm{N}_{\text {ws_01 }}$ & $-0,02 \mathrm{~mm}$ & $-0,01 \mathrm{~mm}$ \\
\hline $\mathrm{E}_{\text {harm_med }}-\mathrm{E}_{\mathrm{ws} \_01}$ & $0,05 \mathrm{~mm}$ & $0,25 \mathrm{~mm}$ \\
\hline $\mathrm{U}_{\text {harm_med }}-\mathrm{U}_{\text {ws_01 }}$ & $-1,01 \mathrm{~mm}$ & $-1,29 \mathrm{~mm}$ \\
\hline
\end{tabular}

Após a determinação dos parâmetros $\mathrm{PCV}_{\text {harm_med }}$ e $\mathrm{PCO}_{\text {harm_med }}$ foi gerado o arquivo em formato de texto para ser aplicado na determinação das coordenadas do pilar 2000 em cada um dos três dias de levantamento. Em seguida, realizou-se a comparação destas coordenadas com as obtidas a partir da aplicação dos parâmetros do programa Wasoft/Kalib.

A Tabela 9 apresenta as diferenças entre as coordenadas do pilar 2000 derivadas da aplicação dos parâmetros $\mathrm{PCV}_{\text {harm_med }}$ e $\mathrm{PCO}_{\text {harm_med }}$ e as coordenadas derivadas da aplicação dos parâmetros $\mathrm{PCV}_{\text {ws_24 }}$ e $\mathrm{PCO}_{\text {ws_24 }}$.

Tabela 9 - Diferenças entre as coordenadas do Pilar 2000 obtidas com parâmetros harm_med e ws_24.

\begin{tabular}{c}
\hline $\begin{array}{c}\text { Diferenças Entre Coordenadas } \\
\text { Pilar 2000 }\end{array}$ \\
\hline$\Delta$ Latitude $=0$ \\
\hline$\Delta$ Longitude $=-1 \times 10^{-5}$, \\
\hline$\Delta$ Altitude $=-2 \times 10^{-4} \mathrm{~m}$ \\
\hline
\end{tabular}

Nota-se que a coordenada longitude obtida com a aplicação dos parâmetros $\mathrm{PCV}_{\text {harm_med }}$ e $\mathrm{PCO}_{\text {harm_med }}$ apresenta diferença de um centésimo de milésimo de segundo de arco quando comparada à coordenada longitude obtida com a aplicação dos parâmetros $\mathrm{PCV}_{\text {ws_24 }}$ e $\mathrm{PCO}_{\text {ws_24. }}$ A coordenada altitude apresenta diferença na ordem de grandeza de décimos de milímetros quando as coordenadas obtidas com aplicação dos parâmetros $\mathrm{PCV}_{\text {harm_med }} \mathrm{e} \mathrm{PCO}_{\text {harm_med }}$ são comparadas às coordenadas obtidas com os parâmetros $\mathrm{PCV}_{\text {ws_24 }}$ e $\mathrm{PCO}_{\text {ws_24 }}$. 
As diferenças entre as coordenadas do ponto 1 obtidas com a aplicação dos parâmetros $\mathrm{PCV}_{\text {harm_med }}$ e $\mathrm{PCO}_{\text {harm_med }}$ e as coordenadas obtidas com a aplicação dos parâmetros $\mathrm{PCV}_{\text {ws_24 }} \mathrm{e} \mathrm{PCO}_{\text {ws_24 }}$ são apresentadas na Tabela 10.

Tabela 3 - Diferenças entre as coordenadas do Ponto 1 obtidas com parâmetros harm_med e ws_24.

\begin{tabular}{c}
\hline $\begin{array}{c}\text { Diferenças Entre Coordenadas } \\
\text { Ponto } 1\end{array}$ \\
\hline$\Delta$ Latitude $=0$ \\
\hline$\Delta$ Longitude $=-1 \times 10^{-5,}$ \\
\hline$\Delta$ Altitude $=-8 \times 10^{-4} \mathrm{~m}$ \\
\hline
\end{tabular}

Percebe-se que a aplicação dos parâmetros harm_med na obtenção das coordenadas do ponto 1 não produz alteração na coordenada altitude. Para a coordenada longitude, a diferença é igual ao centésimo do milésimo de segundo de arco. Na coordenada altitude, a diferença alcança a ordem de grandeza milimétrica, quando comparadas às coordenadas obtidas com a aplicação dos parâmetros ws_24.

A Tabela 11 indica as diferenças algébricas entre as coordenadas do pilar 2000 obtidas com os parâmetros $\mathrm{PCV}_{\text {harm_med }}$ e $\mathrm{PCO}_{\text {harm_med }}$ e obtidas com os parâmetros $\mathrm{PCV}_{\text {ws_27 }}$ e $\mathrm{PCO}_{\text {ws_27. }}$

Tabela 41 - Diferenças entre as coordenadas do Pilar 2000 obtidas com parâmetros harm_med e ws_27.

\begin{tabular}{c}
\hline $\begin{array}{c}\text { Diferenças Entre Coordenadas } \\
\text { Pilar 2000 }\end{array}$ \\
\hline$\Delta$ Latitude $=0$ \\
\hline$\Delta$ Longitude $=1 \times 10^{-5,}$, \\
\hline$\Delta$ Altitude $=-8 \times 10^{-4} \mathrm{~m}$ \\
\hline
\end{tabular}

As diferenças entre as coordenadas do ponto 1 derivadas da aplicação dos parâmetros $\mathrm{PCV}_{\text {harm_med }}$ e $\mathrm{PCO}_{\text {harm_med }}$ e dos parâmetros $\mathrm{PCV}{ }_{\text {ws_27 }}$ e $\mathrm{PCO}_{\text {ws_27 }}$ são indicadas na Tabela 12 .

Tabela 5 - Diferenças entre as coordenadas do Ponto 1 obtidas com parâmetros harm_med e ws_27.

\begin{tabular}{c}
\hline $\begin{array}{c}\text { Diferenças Entre Coordenadas } \\
\text { Ponto 1 }\end{array}$ \\
\hline$\Delta$ Latitude $=0$ \\
\hline$\Delta$ Longitude $=1 \times 10^{-5}$, \\
\hline$\Delta$ Altitude $=-6 \times 10^{-4} \mathrm{~m}$ \\
\hline
\end{tabular}


Nas Tabelas 11 e 12 observa-se que a coordenada latitude permanece inalterada quando são aplicados tanto os parâmetros harm_med quanto os parâmetros ws_27. No entanto, diferenças máximas da ordem de grandeza do centésimo do milésimo de segundo de arco resultam na comparação da coordenada longitude. Com relação à coordenada altitude, as diferenças alcançadas são submilimétricas.

A Tabela 13 apresenta as diferenças entre as coordenadas do pilar 2000 obtidas com os parâmetros harm_med e obtidas com os parâmetros ws_01.

Tabela 6 - Diferenças entre as coordenadas do Pilar 2000 obtidas com parâmetros harm_med e ws_01.

\begin{tabular}{|c|}
\hline $\begin{array}{c}\text { Diferenças Entre Coordenadas } \\
\text { Pilar } 2000 \\
\end{array}$ \\
\hline$\Delta$ Latitude $=0$ \\
\hline$\Delta$ Longitude $=1 \times 10^{-5}$ \\
\hline$\Delta$ Altitude $=1,1 \times 10^{-3} \mathrm{~m}$ \\
\hline
\end{tabular}

A coordenada latitude não sofre alteração diante da aplicação de ambos os conjuntos de parâmetros (harm_med e ws_01). Na coordenada longitude, a diferença apresentada é da ordem de grandeza do centésimo do milésimo de segundo de arco. Para a coordenada altitude, a ordem de grandeza da diferença alcança o patamar milimétrico.

As diferenças algébricas entre as coordenadas do ponto 1 obtidas pela aplicação dos parâmetros $\mathrm{PCV}_{\text {harm_med }}$ e $\mathrm{PCO}_{\text {harm_med }}$ e as coordenadas obtidas pela aplicação dos parâmetros $\mathrm{PCV}_{\text {ws_01 }}$ e $\mathrm{PCO}_{\text {ws_01 }}$ são mostradas na Tabela 14.

Tabela 14 - Diferenças entre as coordenadas do Ponto 1 obtidas com parâmetros harm_med e ws_01.

\begin{tabular}{c}
\hline $\begin{array}{c}\text { Diferenças Entre Coordenadas } \\
\text { Ponto 1 }\end{array}$ \\
\hline$\Delta$ Latitude $=1 \times 10^{-5,}$ \\
\hline$\Delta$ Longitude $=0$ \\
\hline$\Delta$ Altitude $=1,3 \times 10^{-3} \mathrm{~m}$ \\
\hline
\end{tabular}

Nota-se que a diferença para a coordenada latitude é igual ao centésimo do milésimo de segundo de arco quando comparam-se as coordenadas geodésicas derivadas da aplicação dos parâmetros harm_med com as coordenadas derivadas da aplicação dos parâmetros ws_01. Para a coordenada longitude verifica-se que o seu valor não é alterado pela aplicação de ambos os conjuntos de parâmetros. $\mathrm{Na}$ coordenada altitude a diferença algébrica é comparável milímetro. 


\section{CONCLUSÕES}

Percebe-se que a proposta metodológica apresentada produziu resultados promissores uma vez que os coeficientes das funções harmônicas esféricas, considerando $\mathrm{n}=\mathrm{m}=16$, permitiram a estimativa de parâmetros PCV e PCO da antena LEIAX 1202GG, que quando comparados aos parâmetros provenientes do programa de calibração, WaSoft/Kalib, produziram diferenças máximas da ordem do milímetro.

Para cada um dos conjuntos de parâmetros $\mathrm{PCV}$, relativos a cada onda portadora, tomados como dados para a pesquisa - $\mathrm{PCV}_{\text {ws_24 }} \mathrm{e} \mathrm{PCV}_{\text {ws_med }}-$ foram determinados 153 coeficientes $a_{n m}$ e 136 coeficientes $b_{n m}$. Estes produziram novos parâmetros PCV e PCO que na comparação direta com os parâmetros calibrados resultaram em diferenças algébricas máximas de até 6 décimos de milímetros, para as PCVs e 13 décimos de milímetro para a componente vertical do vetor PCO.

Ainda que a proposta metodológica da pesquisa não se caracterize como um método de calibração de antenas, objetiva-se estimar parâmetros compreendidos no âmbito do processo de calibração. Assim, torna-se pertinente mencionar os trabalhos de Görres et al. (2004) e Zeimetz e Kuhlmann (2008), que trazem comparações entre métodos de calibração e os consideram válidos quando diferenças entre os parâmetros dependentes de azimute e ângulo zenital resultam em até $1 \mathrm{~mm}$.

A mudança dos parâmetros do centro de fase da antena e os consequentes resultados das coordenadas geodésicas do pilar 2000 e do ponto 1 sugerem mais investigações dos fatores que afetam o comportamento do centro de fase das antenas GNSS com o objetivo de estimar parâmetros a serem aplicados em eventuais situações em que a calibração não seja possível de ser realizada.

\section{REFERÊNCIAS BIBLIOGRÁFICAS}

ATKINSON, K.E. An introduction to numerical analysis. New York: John Wiley \& Sons, 1978. 693p.

BALANIS, C. A. Antenna theory: analysis and design. New York: John Wiley \& Sons, 2005. 1117p.

FANG, P.; BEVIS, M.; BOCK, Y.; GUTMAN, S.; WOLFE, D. GPS meteorology: reducing systematic errors in geodetic estimates for zenith delay. Geophysics research letters. v. 25, n. 19, p. 3583-3586, 1998.

FREIBERGER JUNIOR, J. Investigação da calibração relativa de antenas GNSS. 2007. 203f. Tese (Doutorado em Ciências Geodésicas) - Curso de PósGraduação em Ciências Geodésicas, Setor de Ciências da Terra, Departamento de Geomática, Universidade Federal do Paraná, Curitiba, 2007.

GÖRRES, B., CAMPBELL, J., SIEMES, M., BECKER, M. New anechoic chamber results and comparison with field and robot techniques. In: IGS WORKSHOP \& SYMPOSIUM, Berne, march 1-5, Switzerland, 2004.

GÖRRES, B.; CAMPBELL, J.; BECKER, M.; SIEMES, M. Absolute calibration of GPS antennas: laboratory results and comparison with field and robot techniques. GPS solutions. v. 10, p. 136-145, 2006. 
HUINCA, S. C. M. Calibração relativa de antenas GNSS na BCAL/UFPR. 2009. 127f. Dissertação (Mestrado em Ciências Geodésicas) - Curso de PósGraduação em Ciências Geodésicas, Setor de Ciências da Terra, Departamento de Geomática, Universidade Federal do Paraná, Curitiba. 2009.

HUINCA, S. C. M.; KRUEGER, C. P.; MAYER, M.; KNÖPFLER, A.; HECK, B. First results of relative field calibration of a GPS antenna at BCAL/UFPR. In: International Association of Geodesy Symposia. Geodesy for planet earth. v. 136, p. 739-744, 2012.

INSTITUTE OF ELECTRICAL AND ELECTRONICS ENGINEERS (IEEE). IEEE standard definitions of terms for antennas. New York, 1983, 29p.

KRAUS, J. D. Antenas. Rio de Janeiro: Guanabara Dois, 1983. 516p.

KRUEGER, C. P.; FREIBERGER JUNIOR, J.; HECK, B.; MAYER, M.; KNÖPFLER, A.; SCHÄFER, B. Establishing a GNSS receiver antenna calibration field in the framework of PROBAL. In: International Association of Geodesy Symposia. Observing our changing earth. v. 133, p. 701-708, 2008.

KRUEGER, C. P.; HUINCA, S. C. M.; LEANDRO, D.; VISKI, A. R. Inovações tecnológicas do laboratório de geodésia espacial e hidrografia (LAGEH). In: IV Simpósio Brasileiro de Ciências Geodésicas e Tecnologias da Geoinformação. Brasil, Recife, 06 - 09 maio, 2012.

MADER, G. GPS antenna calibration at the national geodetic survey. GPS solutions. v. 3, n. 1, p. 50-58, 1999.

MARKOV, G. Antennas. Moscow: Progress Publishers, 1965. 511p.

MENGE, F. Zur kalibrierung der phasenzentrumsvariationen von GPS-antennen für die hochpräzise posistionsbestimmung. 198 f. Genehmigte Dissertation. Hannover, 2003.

MONICO, J. F. G. Posicionamento pelo GNSS: descrição, fundamentos e aplicações. São Paulo: Editora UNESP, 2008. 476 p.

RALSTON, A.; RABINOWITZ, P. A first course in numerical analysis. 2nd edition. New York: McGrawn-Hill, 1984. 556p.

ROTHACHER, M.; SCHAER, S.; MERVAT, L.; BEUTLER, G. Determination of antenna phase center variations using GPS data. In: IGS WORKSHOP, 1995, Potsdam. Proceedings... Potsdam, may 15-17, Germany, 1995.

SCHAER, S.; BEUTLER, G.; MERVAT, L.; ROTACHER, M.; WILD, U. Global and regional ionosphere models using the GPS double difference phase observable. In: IGS WORKSHOP, 1995, Potsdam. Proceedings... Potsdam, may 15-17, Germany, 1995.

SCHMITZ, M; WÜBENNA, G.; BOETTCHER, G. Tests of phase center variations of various GPS antennas, and some results. GPS solutions. v. 6, p.18-27, 2002.

SEEBER, G. Satellite geodesy. 2nd edition. Berlin: De Gruyter, 2003. 589p.

ZEIMETZ, P.; KUHLMANN, H. On the accuracy of absolute GNSS antenna calibration and the conception of a new anechoic chamber. In: GNSS 
ANTENNA CALIBRATION AND ACCURACY ASSESSMENT. FIG WORKING WEEK, Stockholm, june 14-19, Sweden, 2008.

(Recebido em novembro de 2013. Aceito em março de 2014). 